\title{
Consumo de fertilizantes e produtividade da laranja em São Paulo ao longo das décadas de 1970, 1980 e 1990
}

\author{
Margarida Garcia de Figueiredo ${ }^{1}$ \\ Alexandre Lahóz Mendonça de Barros² \\ José Antônio Frizzone ${ }^{3}$
}

Resumo: O Brasil, além de representar sozinho cerca de $80 \%$ do total de suco de laranja concentrado e congelado comercializado no mercado internacional, juntamente com os EUA responde por cerca de $50 \%$ da produção mundial de laranja. O estado de São Paulo, por sua vez, responde por cerca de $80 \%$ da produção nacional de laranja e $98 \%$ das exportações brasileiras de suco concentrado. A relevância econômica dessa atividade tem estimulado inúmeras pesquisas no sentido de analisar diversos aspectos relacionados ao sistema de produção da cultura. Dentre os diversos fatores de produção, o fertilizante é um insumo de grande importância para a cadeia citrícola, especialmente na composição dos custos. Dessa forma, o presente estudo procurou estimar o consumo de fertilizantes pela cultura da laranja em São Paulo ao longo das três últimas décadas. Os resultados encontrados foram satisfatórios, seguindo tendência condizente com a realidade do estado. Também foram desenvolvidos modelos teóricos para estimar o consumo dos nutrientes N, K2O e P2O5, em função da produtividade dos pomares. Finamente, foi desenvolvido um modelo teórico de produtividade (caixas/pé) em função da idade do pomar, baseado em estimativas estocásticas de produtividade para cada idade do pomar. Os modelos ficaram bem ajustados, atendendo a todos os requisitos exigidos do ponto de vista econométrico.

Palavras-chaves: laranja, fertilizantes, produtividade, São Paulo, modelo econométrico.

\footnotetext{
${ }^{1}$ Professora na Faculdade de Economia da Universidade Federal de Mato Grosso (UFMT).E-mail: margaridagf@ufmt.br

2 Professor da Escola de Economia de São Paulo da Fundação Getúlio Vargas (FGV). E-mail: almb@fgvsp.br

${ }^{3}$ Departamento de Engenharia Rural da Escola Superior de Agricultura Luiz de Queiróz (Esalq), USP. E-mail: frizzone@esalq.usp.br
} 


\begin{abstract}
Brazil represents approximately $80 \%$ of the total frozen concentrate orange juice (FCOJ) traded in the international market. In addition, Brazil and the United States represent $50 \%$ of the total fresh orange produced around the world. Sao Paulo state is responsible by $98 \%$ of Brazilian FCOJ exports and by $80 \%$ of the national fresh orange production. The economic importance of Sao Paulo's citrus industry has been stimulated researches in order to analyze all aspects related to citrus production system. Fertilizer is a very important input into citrus industry, especially in relation to production costs. Thus, this study has outlined the fertilizer consumed by Sao Paulo's citrus industry in the 70's, 80 's and 90's. It was developed models to estimate fertilizer consumption related to orange trees yields. Finally, it was developed a model to estimate an orange tree yield (boxes/tree) in relation to grove age from stochastic yields values estimated to each tree age. The models were well adjusted and meeting all econometrics requisites.
\end{abstract}

Key-words: orange, fertilizer, yield, Sao Paulo, econometric model.

Classificação JEL: C1, C13.

\title{
1. Introdução
}

O Brasil, além de representar sozinho cerca de $80 \%$ do total de suco de laranja concentrado e congelado comercializado no mercado internacional, juntamente com os Estados Unidos, responde por cerca de 50\% da produção mundial de laranja, segundo estatísticas da Food and Agriculture Organization of United Nations (FAO, 2005). O estado de São Paulo, por sua vez, responde por cerca de $80 \%$ da produção nacional de laranja e $98 \%$ das exportações brasileiras de suco concentrado, de acordo com estatísticas da Associação Brasileira dos Exportadores de Citros (ABECITRUS, 2005).

A relevância econômica dessa atividade tem estimulado inúmeras pesquisas no sentido de aprimorar as técnicas de produção na cultura, desde o plantio até a colheita das frutas. Em geral, a evolução das técnicas tende a reduzir os gastos com mão-de-obra e aumentar o emprego de capital em máquinas e implementos agrícolas. Por outro lado, o uso de fertilizantes e defensivos se associa às práticas exigentes em trabalho mais qualificado e aumento da produtividade.

De acordo com Neves et al. (2005), o fertilizante é um insumo de grande importância para a cadeia citrícola, principalmente na composição do custo da atividade. Segundo estatísticas da Associação Nacional para Difusão de Adubos (Anda), o consumo de fertilizantes pela laranja no Brasil cresceu 17\% de 2000 a 2004 , totalizando 425 mil toneladas em 2005. Diante da importância do fertilizante no sistema de produção da laranja e, considerando que a produtividade depende, entre outros fatores, de um adequado manejo da adubação, torna-se interessante a realização de estudos que procurem analisar as relações entre consumo de 
fertilizantes, produtividade e idade dos pomares no estado de São Paulo. Vale ressaltar que não se encontra disponível na literatura estatística completa a respeito do consumo de fertilizantes pela citricultura paulista ao longo dos últimos 35 anos.

Dessa forma, o objetivo deste estudo foi estimar o consumo de fertilizantes pelo setor citrícola (especificamente pela cultura da laranja) no estado de São Paulo desde 1970 até 2005, bem como analisar a variação na quantidade aplicada de fertilizantes de acordo com a produtividade do pomar. Além disso, foi desenvolvido um modelo econométrico para estimativa da produtividade (caixas de 40,8 kg / pé) em função da idade do pomar, partindo-se dos valores esperados de produtividade para cada idade do pomar entre 3 e 20 anos. Os valores esperados foram estimados a partir de técnicas especiais de simulação (métodos de Hertz e de Monte Carlo), cujos dados de entrada foram produtividades de 43 diferentes talhões localizados no estado de São Paulo.

\section{Metodologia}

Para a estimativa do consumo de fertilizantes pela laranja em São Paulo, partiu-se do consumo de fertilizantes pela laranja no Brasil, disponível no Anuário Estatístico do Setor de Fertilizantes da Anda, de 1986 até 2004. Para este período, multiplicou-se o consumo de fertilizantes pela laranja no Brasil, pela participação de São Paulo sobre a área colhida com laranja no Brasil:

$$
\text { CFLSP }_{i}=\text { CFLB }_{i} *\left(\frac{A L S P_{i}}{A L B_{i}}\right), i=1986, \ldots, 2004
$$

Em que:

CFLSP $_{i}=$ Consumo de fertilizantes pela laranja em São Paulo no ano $i$

$C F L B_{i}=$ Consumo de fertilizantes pela laranja no Brasil no ano $i$

$A L S P_{i}=$ Área colhida com laranja em São Paulo no ano $i$

$A L B_{i}=$ Área colhida com laranja no Brasil no ano $i$

Uma vez obtido o consumo de fertilizantes pela laranja em São Paulo, dividiu-se este valor pelo número total de pés de laranja no estado de São Paulo, fornecido pelo Instituto de Economia Agrícola (IEA), obtendo, assim, o consumo de fertilizantes por pé de laranja ao longo do período:

$$
\text { CFPLSP }_{i}=\left(\frac{\text { CFLSP }_{i}}{\text { NPLSP }_{i}}\right), i=1986, \ldots, 2004
$$

Em que:

CFPLSP $_{i}=$ Consumo de fertilizantes por pé de laranja em São Paulo no ano $i$

CFLSP $_{i}=$ Consumo de fertilizantes pela laranja em São Paulo no ano $i$

$N P L S P_{i}=$ Número de pés de laranja em São Paulo no ano $i$ 
Analisando-se o consumo de fertilizantes por pé de laranja entre 1986 e 2004, verificou-se que não houve grandes variações ao longo dos anos e, portanto, calculou-se a média de consumo por pé ao longo do período. Ao multiplicar esta média pelo número total de pés de laranja no estado de São Paulo a cada ano, estimou-se o consumo de fertilizantes pela laranja, entre 1970 e 1985. Assim, obteve-se a série completa de consumo de fertilizantes pela laranja em São Paulo, de 1970 a 2004.

Com relação à variação na quantidade aplicada de fertilizantes, baseado nos dados obtidos em estudo desenvolvido por Dias et al. (2002), estimaram-se os parâmetros para determinar a quantidade aplicada de $\mathrm{N}, \mathrm{K}_{2} \mathrm{O}$ e $\mathrm{P}_{2} \mathrm{O}_{5}$, em função da produtividade do pomar, a partir do seguinte modelo teórico:

$$
Y=a+b X^{*} \ln (X)
$$

Em que:

$Y=$ Consumo de $\mathrm{N}, \mathrm{K}_{2} \mathrm{O}$ ou $\mathrm{P}_{2} \mathrm{O}_{5}(\mathrm{em} \mathrm{kg} / \mathrm{ha})$

$X=$ Produtividade do pomar (em $\mathrm{t} / \mathrm{ha})$

Além do consumo de fertilizantes, do clima, do tipo de solo, da combinação entre copa e porta-enxerto, dentre outros fatores, a idade das árvores afeta consideravelmente a produtividade de um pomar de laranja. Por esta razão, estimou-se, também, um modelo teórico de produtividade (em caixas de 40,8 kg por pé) em função da idade do pomar. Devido à grande variabilidade observada dentre os diversos talhões localizados em diferentes regióes no parque citrícola estadual, optou-se por estimar o modelo a partir dos valores esperados de produtividade para cada idade do pomar. Tais valores esperados foram estimados utilizando-se dois diferentes métodos de simulação (Método de Hertz e Método de Monte Carlo), cujos valores iniciais foram obtidos a partir de valores de produtividade para 43 diferentes talhões localizados no estado de São Paulo. Uma vez de posse dos dados, o primeiro passo foi fazer um teste de aderência (Qui-quadrado $-\chi^{2}$ ) para identificação das distribuições de probabilidades que melhor se ajustassem aos mesmos. Verificou-se que, para as árvores entre 7 e 20 anos de idade, os dados apresentaram um bom ajuste à distribuição normal de probabilidades e, portanto, utilizou-se o método de Monte Carlo (para a distribuição normal) na simulação dos valores de produtividade. Nos demais casos (árvores de 3 a 6 anos de idade), em que os dados não se ajustaram bem à distribuição normal, as simulações foram realizadas pelo Método de Hertz, com distribuições empíricas. Em todos os casos foram gerados 2.000 valores aleatórios para simulação dos resultados. 


\section{Método de Monte Carlo para simulação}

O método de Monte Carlo é uma técnica de gerar informações por meio de simulação, utilizando-se números aleatórios. O método explora as propriedades estatísticas dos números aleatórios, assegurando que cada resultado a ser obtido tenha a mesma probabilidade de ocorrência. A precisão do resultado final depende, em geral, do número de tentativas, em outras palavras, da quantidade de números aleatórios considerados na simulação.

De acordo com Frizzone et al. (2005), considerando a distribuição normal, uma vez que um decimal aleatório tem uma distribuição uniforme de zero a um, ele tem média $1 / 2$ e desvio padrão $1 / \sqrt{2}$. Esse teorema implica que a soma de $n$ números decimais aleatórios tenha, aproximadamente, distribuição normal com média $n / 2$ e desvio padrão $\sqrt{n / 12}$. Portanto, se $r_{1}, r_{2}, \ldots, r_{n}$ forem uma amostra de números decimais aleatórios, então:

$$
X=\frac{\sigma}{\sqrt{\frac{n}{12}}} \sum_{i=1}^{n} r_{i}+\left(\mu-\frac{n}{2} \frac{\sigma}{\sqrt{\frac{n}{12}}}\right)
$$

É uma observação aleatória a partir de uma distribuição normal com média $\mu$ e desvio padrão $\sigma$. Essa aproximação é adequada, exceto nas pontas da distribuição, mesmo com valores pequenos de $n$. Por isso, $n=12$ é um valor conveniente, pois elimina os termos de raiz quadrada da equação (04). Para $n=12$, tem-se:

$$
X=\sigma \sum_{i=1}^{n} r_{i}+(\mu-6 \sigma)
$$

\section{Método de Hertz para simulação}

De acordo com Frizzone et al. (2005), a partir de uma série de valores, associados às suas respectivas freqüências acumuladas, ajusta-se uma função interpoladora para números aleatórios, a partir da qual gera-se uma seqüência de valores para a variável de estudo.

Função interpoladora para árvores entre 3 e 6 anos de idade:

$$
Y=a^{*} \exp (-X / b)
$$

Em que:

$Y=$ Produtividade do pomar em caixas por pé

$X=$ Probabilidade (acumulada) de ocorrência de cada valor de produtividade 
Após estimar as produtividades esperadas para cada idade do pomar, utilizando-se os dois métodos de simulação descritos anteriormente, o próximo passo foi estimar os parâmetros para determinar a produtividade em função da idade do pomar, a partir da função Log-Normal.

Modelo teórico da produtividade em função da idade (Função Log-Normal):

$Y=a^{*} \exp \left[-0,5^{*}\left(\frac{\ln (X / b)}{c}\right)^{2}\right]$

Em que:

$Y=$ Produtividade esperada do pomar em caixas por pé

$X=$ Idade do pomar

\section{Resultados e Discussão}

A média de consumo de fertilizantes por pé de laranja no estado de São Paulo, entre 1986 e 2005, foi de 1,34 kg. Observando a Figura 1, a qual mostra a tendência do consumo ao longo do período analisado, verifica-se uma queda no consumo por pé de 1989 a 1991 e, a partir daí, este permaneceu relativamente constante. Esta queda no consumo de fertilizantes por pé de laranja pode estar associada a alterações na tecnologia de produção, ao melhoramento genético, dentre outros fatores.

Figura 1. Índice de variação do consumo de fertilizantes (em toneladas) por pé de laranja em São Paulo, de 1986 a 2004 (1986=100).

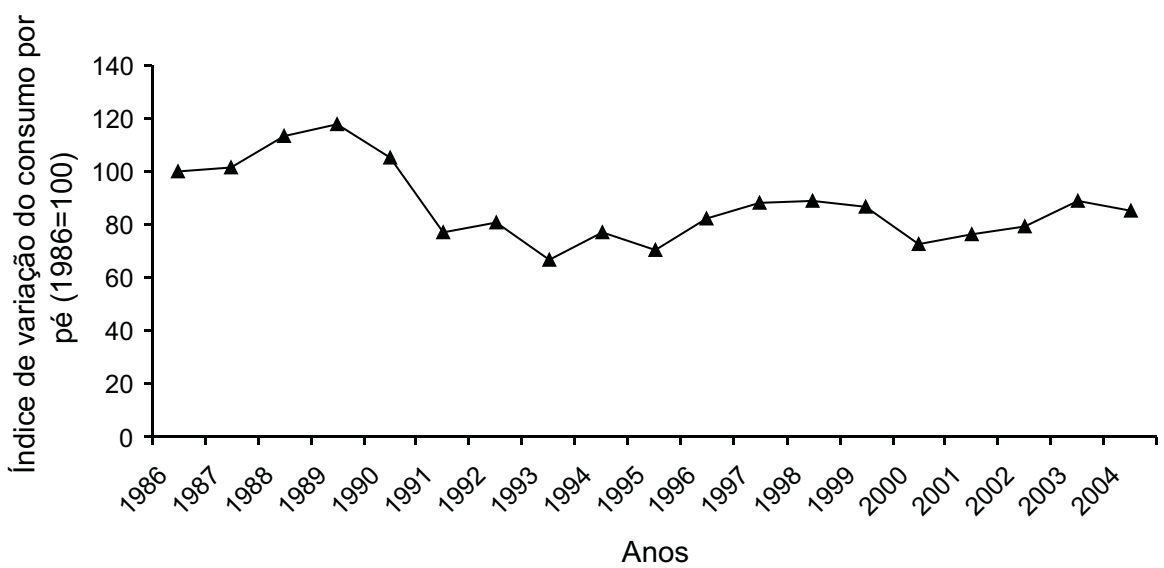

Fonte: Anda. 
A Tabela 1 apresenta os valores do consumo de fertilizantes pela citricultura (especificamente para a laranja) no estado de São Paulo entre 1970 e 2005.

Tabela 1. Consumo de fertilizantes (toneladas) pela laranja no estado de São Paulo (1970-2005).

\begin{tabular}{cccccc}
\hline Ano & Consumo & Ano & Consumo & Ano & Consumo \\
\hline 1970 & 52.083 & 1982 & 143.670 & 1994 & 262.774 \\
1971 & 58.761 & 1983 & 148.809 & 1995 & 252.970 \\
1972 & 67.041 & 1984 & 156.641 & 1996 & 285.096 \\
1973 & 81.464 & 1985 & 174.641 & 1997 & 300.533 \\
1974 & 100.962 & 1986 & 206.692 & 1998 & 305.579 \\
1975 & 101.229 & 1987 & 225.005 & 1999 & 302.485 \\
1976 & 109.509 & 1988 & 271.816 & 2000 & 239.115 \\
1977 & 106.504 & 1989 & 311.841 & 2001 & 239.027 \\
1978 & 119.577 & 1990 & 291.358 & 2002 & 256.347 \\
1979 & 137.914 & 1991 & 232.768 & 2003 & 290.685 \\
1980 & 142.335 & 1992 & 253.572 & 2004 & 280.676 \\
1981 & 141.880 & 1993 & 219.097 & 2005 & 303.842 \\
\hline
\end{tabular}

Fonte: Estimativa (1970-1985) e Anda (1986-2004).

A Figura 2 permite visualizar o comportamento deste consumo ao longo do período. É interessante observar a acentuada tendência de aumento no consumo de fertilizantes pelo setor citrícola entre o final da década de 1970 e o início da década de 1990. Tal crescimento pode ser justificado tanto pelo adensamento do plantio, possibilitando a produção de um maior número de caixas de laranja por hectare (o que representa um ganho de produtividade parcial), quanto pelo aumento na área cultivada com laranja em São Paulo. De acordo com estatísticas do IBGE, a área cultivada com laranja no estado paulista apresentou um aumento de 545\% (ou seja, aumentou em mais de seis vezes) entre 1970 e 1990, conforme verificado na Figura 3.

No que diz respeito ao consumo de fertilizantes pela cultura, as recomendações de adubação para laranja se dividem em adubação de plantio, adubação de formação (do primeiro ao quarto ano) e adubação de produção (do quinto ano em diante). As recomendações nos dois primeiros casos costumam indicar uma dosagem de fertilizante baseado na análise de solo. No caso da adubação de produção, os critérios de recomendação se baseiam em diversos aspectos, principalmente na análise de solo, na análise foliar (para identificar a necessidade da planta) e na produtividade do pomar. Dessa forma, o presente estudo estima alguns parâmetros para modelos teóricos de determinação do consumo de $\mathrm{N}, \mathrm{K}_{2} \mathrm{O}$ e $\mathrm{P}_{2} \mathrm{O}_{5}$, em função da produtividade do pomar. Do ponto de vista econométrico, os parâmetros estimados foram os que melhor se ajustaram a todos os requisitos exigidos, conforme exposto na Tabela 2. 
Figura 2. Índice de variação do consumo de fertilizantes (em toneladas) pela laranja em São Paulo, de 1970 a $2005(1970=100)$.

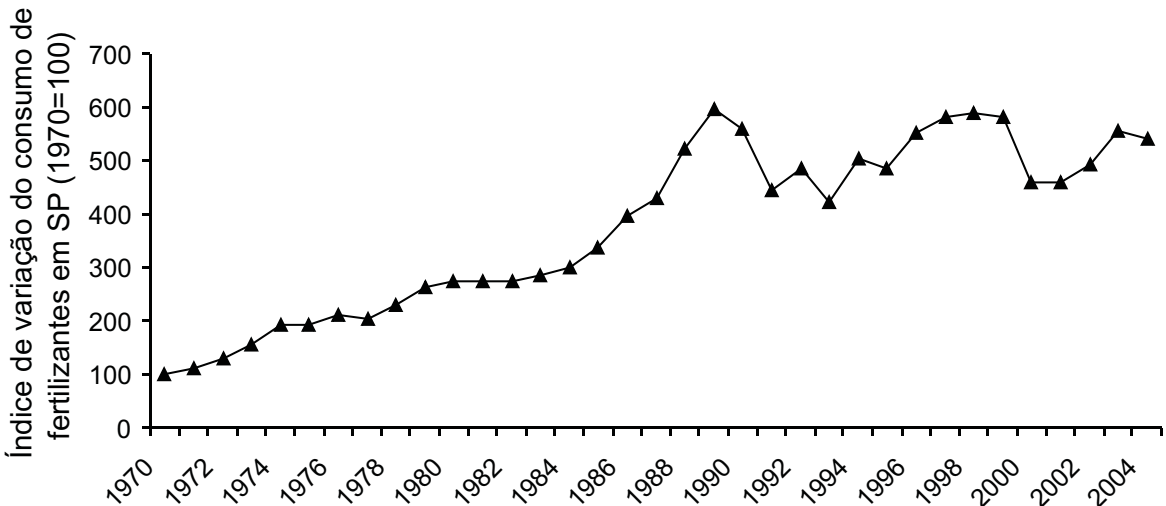

Anos

Fonte: Estimativa (1970-1985) e Anda (1986-2005).

Figura 3. Índice de variação da área cultivada com laranja em São Paulo, de 1970 a $2005(1970=100)$.

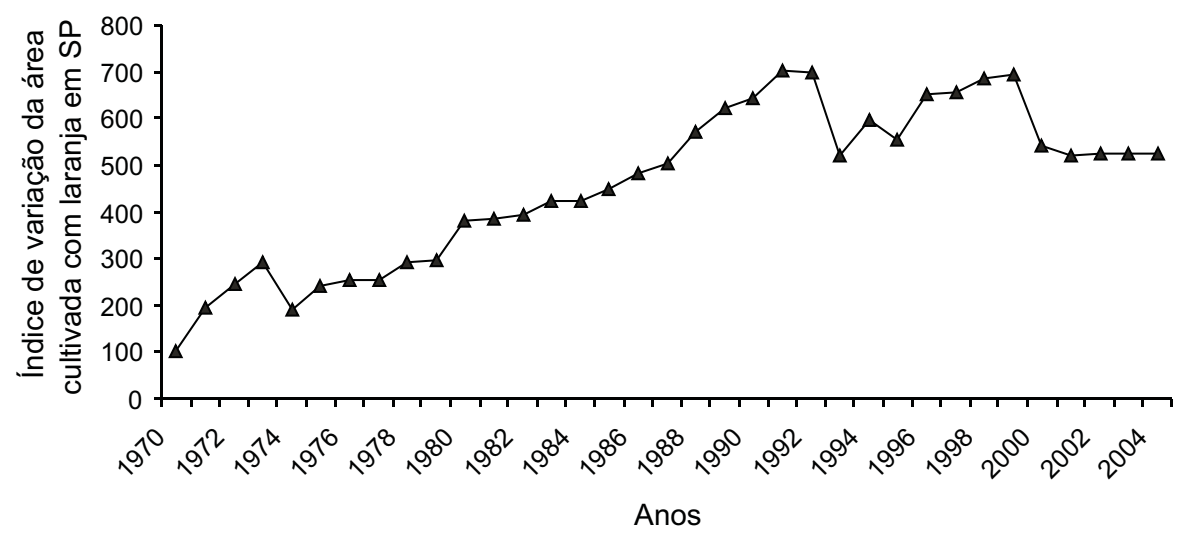

Fonte: IBGE.

Tabela 2. Estimativas dos parâmetros dos modelos para consumo de fertilizantes em função da produtividade do pomar.

\begin{tabular}{ccccccc}
\hline \multirow{2}{*}{ Parâmetros } & \multicolumn{2}{c}{ Nitrogênio $(\mathbf{N})$} & \multicolumn{2}{c}{ Potássio $\left(\mathrm{K}_{2} \mathbf{O}\right)$} & \multicolumn{2}{c}{ Fósforo $\left(\mathbf{P}_{2} \mathbf{O}_{5}\right)$} \\
\cline { 2 - 7 } & Valor & Teste $\mathbf{t}$ & Valor & Teste $\mathbf{t}$ & Valor & Teste $\mathbf{t}$ \\
\hline $\mathrm{a}$ & 21,14 & $7,59^{*}$ & 15,75 & $21,89^{*}$ & 14,53 & $13,69^{*}$ \\
$\mathrm{~b}$ & 1,05 & $63,17^{*}$ & 0,80 & $231,87^{*}$ & 0,76 & $166,35^{*}$ \\
\hline
\end{tabular}

Nota: *denota significância a $1 \%$ 
As Figuras 4, 5 e 6 ilustram o consumo (observado e estimado) de N, $\mathrm{K}_{2} \mathrm{O}$ e $\mathrm{P}_{2} \mathrm{O}_{5}$, respectivamente, para cada nível de produtividade de um pomar de laranja.

Figura 4. Consumo de $\mathrm{N}$ em função da produtividade do pomar.

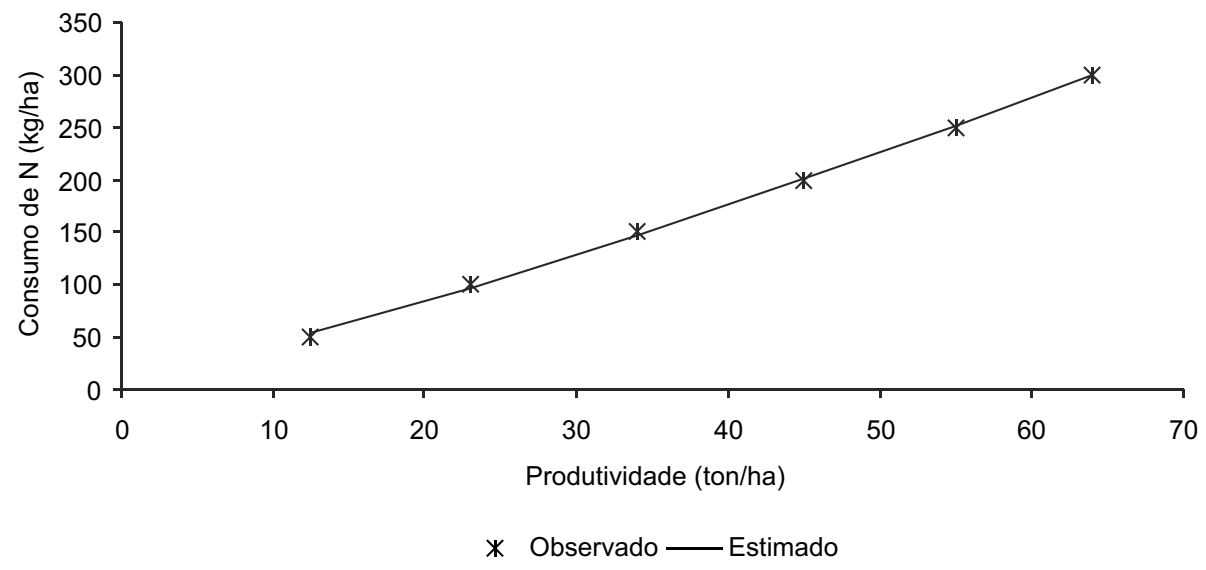

Fonte: Estimativa a partir de dados disponibilizados em Dias et al. (2002).

Figura 5. Consumo de $\mathrm{K}_{2} \mathrm{O}$ em função da produtividade do pomar.

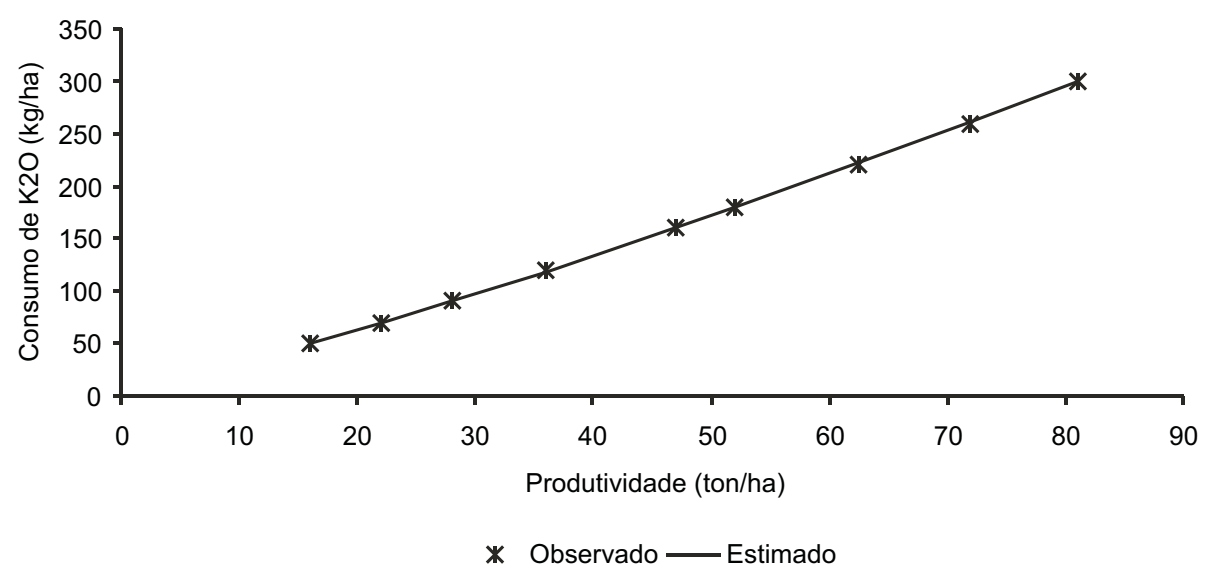

Fonte: Estimativa a partir de dados disponibilizados em Dias et al. (2002). 
Figura 6. Consumo de $\mathrm{P}_{2} \mathrm{O}_{5}$ em função da produtividade do pomar.

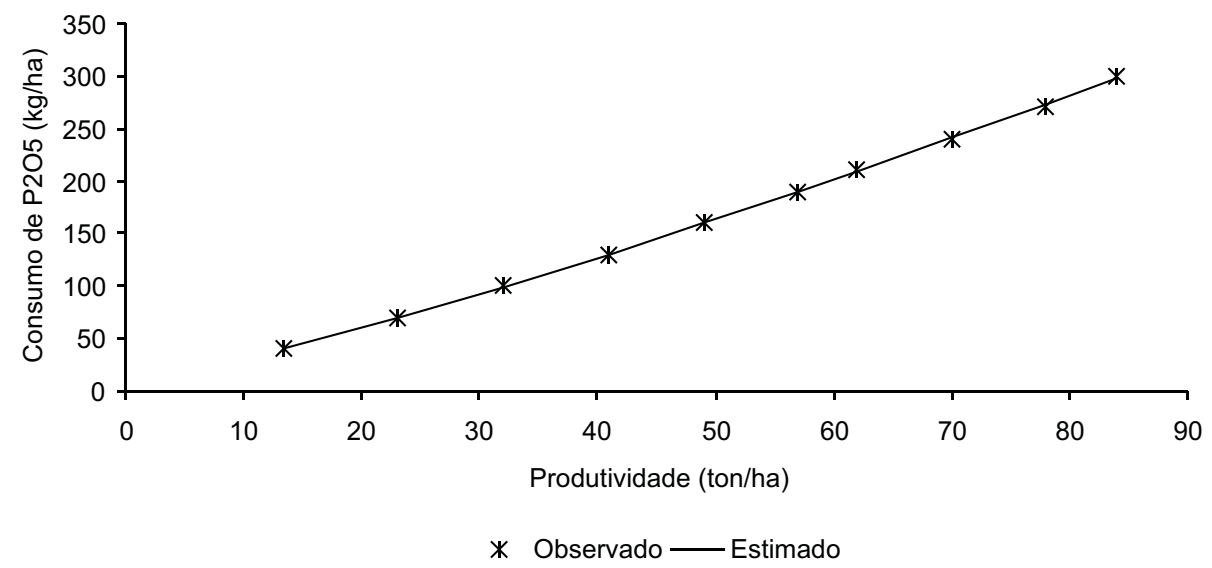

Fonte: Estimativa a partir de dados disponibilizados em Dias et al. (2002).

Quanto às estimativas dos parâmetros das funções para simulação de produtividade (função interpoladora para números aleatórios), no caso das árvores entre 3 e 6 anos de idade, os valores encontram-se expostos na Tabela 3. Do ponto de vista econométrico, os parâmetros estimados foram os que melhor se ajustaram a todos os requisitos exigidos.

Tabela 3. Estimativas dos parâmetros dos modelos para simulação de produtividade do pomar para árvores entre 3 e 6 anos de idade.

\begin{tabular}{ccccccccc}
\hline & \multicolumn{2}{c}{3 anos } & \multicolumn{2}{c}{4 anos } & \multicolumn{2}{c}{5 anos } & \multicolumn{2}{c}{6 anos } \\
\cline { 2 - 9 } & Valor & Teste $\mathbf{t}$ & Valor & Teste $\mathbf{t}$ & Valor & Teste $\mathbf{t}$ & Valor & Teste $\mathbf{t}$ \\
\hline a & 0,02 & $2,65^{* *}$ & 0,08 & $6,63^{*}$ & 0,13 & $3,12^{* *}$ & 0,55 & $3,32^{* *}$ \\
b & $-0,25$ & $-9,87^{*}$ & $-0,33$ & $-18,67^{*}$ & $-0,35$ & $-8,30^{*}$ & $-0,66$ & $-4,55^{*}$ \\
\hline
\end{tabular}

Nota: *denota significância a $1 \%,{ }^{* *}$ denota significância a $5 \%$.

No caso das árvores entre 7 e 20 anos de idade, as produtividades esperadas foram simuladas pelo método de Monte Carlo para distribuição normal, não tendo sido necessário, portanto, a estimativa de função interpoladora para números aleatórios. Os resultados de produtividades esperadas por idade da árvore, utilizados como dados de entrada para estimativa dos parâmetros do modelo teórico de produtividade em função da idade, encontram-se expostos na Tabela 4. 
Tabela 4. Produtividade esperada (caixas/pé) para cada idade da árvore.

\begin{tabular}{cccc}
\hline Idade & $\begin{array}{c}\text { Produtividade } \\
\text { (caixas/pé) }\end{array}$ & Idade & $\begin{array}{c}\text { Produtividade } \\
\text { (caixas/pé) }\end{array}$ \\
\hline 3 anos & 0,22 & 12 anos & 2,84 \\
4 anos & 0,51 & 13 anos & 2,87 \\
5 anos & 0,72 & 14 anos & 2,86 \\
6 anos & 1,29 & 15 anos & 2,84 \\
7 anos & 1,83 & 16 anos & 2,68 \\
8 anos & 2,29 & 17 anos & 2,67 \\
9 anos & 2,70 & 18 anos & 2,52 \\
10 anos & 2,72 & 19 anos & 2,46 \\
11 anos & 2,81 & 20 anos & 2,21 \\
\hline
\end{tabular}

Fonte: Simulação a partir de dados observados para 43 talhões no estado de São Paulo.

Verificou-se que, a partir dos três anos de idade, o pomar começa a produzir, apresentando maior produtividade, até atingir o valor máximo em torno dos 13 anos, começando a decrescer a partir daí. Esta perda de produtividade à medida que o pomar vai ficando mais velho pode estar relacionada a uma série de fatores, destacando-se entre eles os aspectos fitossanitários, o principal problema enfrentado pela citricultura atualmente.

Do ponto de vista econométrico, os parâmetros estimados para o modelo Log-Normal foram os que melhor se ajustaram aos dados de produtividade em função da idade do pomar, sendo todos significativos a $1 \%$ de acordo com o Teste $\mathrm{t}$, conforme exposto na Tabela 5 .

Tabela 5. Estimativas dos parâmetros do modelo Log-Normal para determinação da produtividade em função da idade do pomar.

\begin{tabular}{ccc}
\hline Parâmetros & Valor & Teste t \\
\hline a & 2,94 & $122,68^{*}$ \\
b & 12,73 & $113,82^{*}$ \\
c & 0,61 & $53,52^{*}$ \\
\hline
\end{tabular}

Nota: *denota significância a $1 \%$

$$
Y=2,94^{*} \exp \left[-0,5 *\left(\frac{\ln (X / 12,73)}{0,61}\right)^{2}\right]
$$


Substituindo os valores de idade das árvores em $X$, foi possível estimar a produtividade da árvore $(\mathrm{Y})$ a cada idade, conforme visualizado na Figura 7 abaixo.

Figura 7. Produtividade (em caixas/pé) em função da idade.

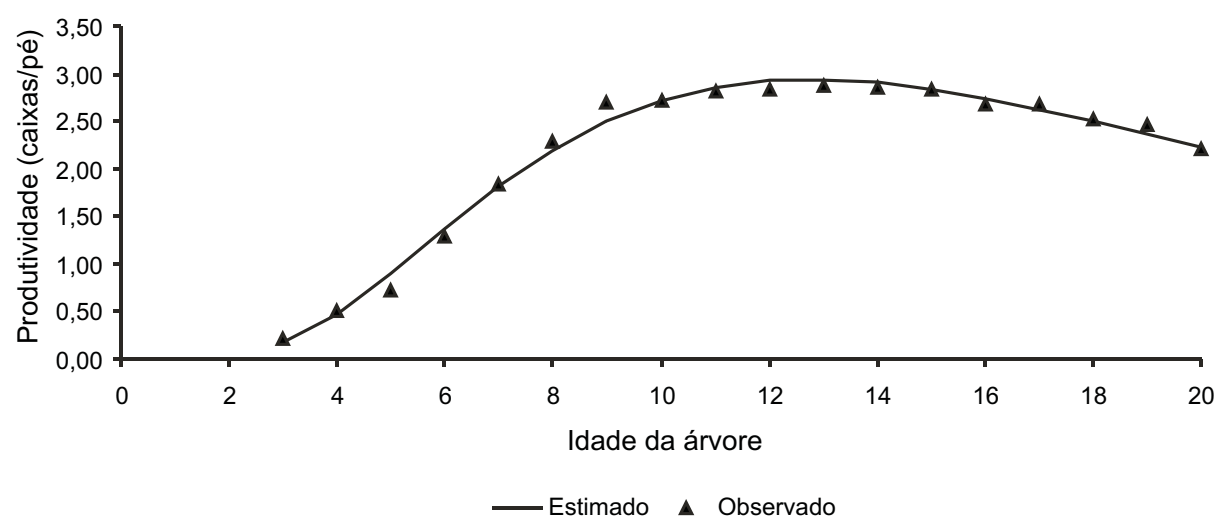

Fonte: Talhões de laranja no estado de São Paulo (os dados observados se referem às produtividades esperadas para cada idade da árvore).

Ao se confrontar os modelos de produtividade em função da idade com os modelos de consumo de fertilizantes em função da produtividade, é possível identificar qual dosagem de fertilizantes $\left(\mathrm{N}, \mathrm{P}_{2} \mathrm{O}_{5}\right.$ e $\left.\mathrm{K}_{2} \mathrm{O}\right)$ mais apropriada para cada idade do pomar, conforme verificado na Figura 8.

Figura 8. Consumo de fertilizantes de acordo com a idade do pomar.

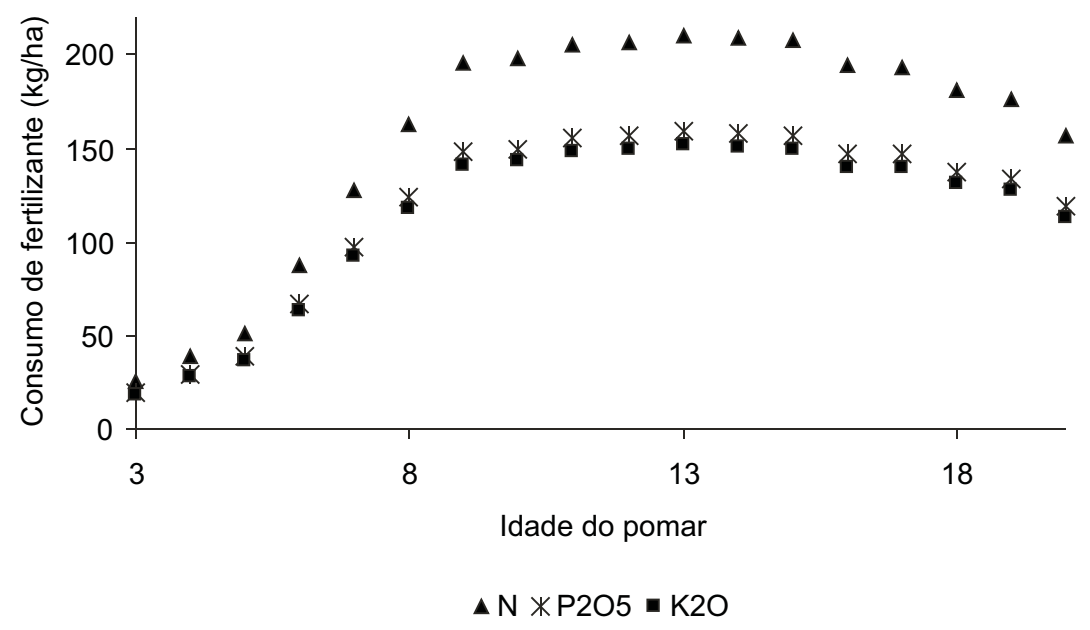




\section{Conclusão}

Em vista da importância que tem a citricultura para o Brasil e, em particular para o estado de São Paulo, e considerando a importância do fertilizante nesta atividade, o presente estudo procurou estimar o consumo de fertilizantes pela cultura da laranja em São Paulo ao longo das três últimas décadas. Os resultados encontrados foram satisfatórios, seguindo tendência condizente com a realidade do estado. Houve um aumento no consumo de fertilizantes entre as décadas de 1970 e 1990, que se justificam tanto pelo adensamento do plantio quanto pelo aumento na área cultivada com laranja em São Paulo.

Além disso, foram estimados alguns parâmetros de modelos teóricos para determinação tanto do consumo dos nutrientes $\mathrm{N}, \mathrm{K}_{2} \mathrm{O}$ e $\mathrm{P}_{2} \mathrm{O}_{5}$ em função da produtividade dos pomares, quanto da produtividade em função da idade do pomar. Os modelos ficaram bem ajustados, de modo que todos os parâmetros estimados atenderam a todos os requisitos exigidos do ponto de vista econométrico. Verificou-se que tanto a produtividade (em caixas/pé) quanto o consumo de fertilizantes (em toneladas/pé) apresentaram um comportamento crescente até atingirem os seus valores máximos ao redor dos 13 anos de idade da árvore e, depois, passaram a cair.

\section{Referências Bibliográficas}

ASSOCIAÇÃO BRASILEIRA DOS EXPORTADORES DE CITROS. ABECITRUS: exportações de FCOJ - série histórica. Disponível em http://www.abecitrus.com.br/exporta_sh_br.html>. Acesso em: 4 jul. 2005.

ANUÁRIO ESTATÍSTICO DO SETOR DE FERTILIZANTES vários anos, São Paulo, Associação Nacional para Difusão de Adubos e corretivos Agrícolas (ANDA).

BRASIL. Ministério do Desenvolvimento, Indústria e Comércio Exterior. Secretaria de Comércio Exterior. Disponível em: <http://www.desenvolvimento.gov.br/ sitio/secex/depPlaDesComExterior/indEstatisticas/balComercial.php $>$. Acesso em: 20 out. 2005.

BRIEGER, F.G; MOREIRA, S.; LEME, Z. Estudo sobre o melhoramento da laranja Bahia: III. Bragantia, v1, p. 567-610, 1941.)

FOOD AND AGRICULTURE ORGANIZATION OF UNITED NATIONS. Statistical databases. FAOSTAT: agriculture. Disponível em : $<$ http://www.fao.org >. Acesso em: 1 fev. 2005. 
FRIZZONE, J. A.; ANDRADE JÚNIOR, A. S. (Ed.). Planejamento da irrigação: análise de decisão de investimento. Brasília, DF: Embrapa Informação Tecnológica; Teresina: Embrapa Meio Norte, 2005. 627p.

INSTITUTO BRASILEIRO DE GEOGRAFIA E ESTATÍSTICA. Banco de dados agregados. SIDRA: Produção Agrícola municipal. Disponível em : <http://www.ibge.gov.br>. Acesso em: 2 dez. 2004.

INSTITUTO DE ECONOMIA AGRÍCOLA. Banco de dados. Disponível em: http://www.iea.sp.gov.br. Acesso em: 4 fev. 2006.

MOREIRA, S. Observações sobre a tristeza dos citros ou podridão das radicelas. $\mathbf{O}$ Biológico. São Paulo, n.11, p.269-272, 1942.

NEVES, M.F.; LOPES, F.F. Estratégias para laranja no Brasil. 1.ed. São Paulo: Atlas, 2005. $225 \mathrm{p}$.

RODRIGUES, O.; VIEGAS, F.; POMPEU Jr., J; AMARO, A.A. Citricultura brasileira - Segunda Edição, Volume 1. Fundação Cargill, Campinas, 1991.491 p.

ROSSETTI, V.; SALIBE, A.A.; CINTRA, A.F.; BONILHA, S. ARMBRUSTER, D. The citrus budwood certification program in the State of São Paulo. In: PRICE, W.C. ed. CONF. of INTER. ORGANIZATION CITRUS VIROL., 3., Fla., 1965. Proceedings. Gainesville, Univ. Gainesville, Fla. Press, 1965. p.235-240 